\title{
GaN and InGaN Nanowires on Si Substrates by Ga-Droplet Molecular Beam Epitaxy
}

Kevin Goodman, Kejia Wang, Xiangning Luo, John Simon, Tom Kosel, and Debdeep Jena Electrical Engineering, University of Notre Dame, 275 Fitzpatrick Hall, Notre Dame, IN, 46556

\section{ABSTRACT}

Molecular beam epitaxy growth of $\mathrm{GaN}$ and $\mathrm{InGaN}$ nanowires is accomplished on $\mathrm{Si}$ (111) substrates using Ga-droplet nucleation. Typical diameters range from 25-80 nm and lengths can be varied by increasing the growth time; the growth rate is $\sim 0.25$ microns/hour. The nanowires have been characterized structurally and optically. Photoluminescence spectra show band-edge emission of GaN nanowires centered at $362 \mathrm{~nm}$ at $290 \mathrm{~K}$. Transmission electron microscopy images unveil that the nanowires are highly crystalline, and grow along the 0001 polar direction. Indium has also been successfully incorporated into $\mathrm{GaN}$ nanowires by modifying the growth conditions; the InGaN nanowires emit at $\sim 520 \mathrm{~nm}$, which provides a possible route to solving strain related problems of high In-composition InGaN based efficient green emitters.

\section{INTRODUCTION}

Today's world is seeing an ever increasing cost of energy with no downturn in sight for the immediate future [1]. Therefore it is only logical to look for every possible method to conserve energy. One means to this end is to replace conventional lighting such as incandescent or fluorescent lamps with light emitting diodes which offer higher luminescent efficiencies and longer lifetimes. Light emitting diodes (LEDs) producing white light are possible by combining blue, red, and green wavelength light sources [2]. The III-V Nitride semiconductor family (InN, $\mathrm{GaN}$, and AlN) spans this entire wavelength range. Therefore, by altering the compositions of ternary InGaN materials, all three wavelengths needed to construct white light sources can be produced from the single material family. Currently, the green region of the spectrum (specifically the $520-580 \mathrm{~nm}$ window) is not adequately covered by nitride LEDs, due to the low crystalline quality of high-Indium composition InGaN layers.

A problem that surfaces in growing c-plane high In-composition InGaN 2D layers on $\mathrm{GaN}$ substrates is the considerable lattice mismatch between $\mathrm{InN}$ (c-lattice constant $0.353 \mathrm{~nm}$ ), and the substrate $(\mathrm{GaN}, 0.319 \mathrm{~nm})$. This difference results in high strain in the $\mathrm{InGaN}$, resulting in the formation of dislocations and other defects in thick layers. These defects act to introduce recombination centers and electronic traps which lower the optical emission efficiency of light emitting devices. Recent research has shown [3] that one method to circumvent these problems is to grow non-2D structures, specifically pillars or 1D-nanowires of InGaN. Since the nanowires in principle are free to expand or contract to their desired inherent lattice constants in the growth planes, considerable (and perhaps complete) strain relaxation can be achieved in this fashion. This offers crystalline materials free of defects, which has the potential to increase the optical emission efficiency of the material. Though major challenges such as n- and p-type doping, formation of electrical contacts to nanowire tips, control of surface recombination, and efficient light extraction from nanowire based LEDs are anticipated in the future, the first step in this endeavour is the demonstration of the growth of defect-free high-In composition InGaN nanowires that emit in the $520-580 \mathrm{~nm}$ window. 
Growth of nitride nanowires has been achieved by both Metal-Organic Chemical Vapor Deposition (MOCVD) and Molecular Beam Epitaxy (MBE) [4, 5]. Due to very efficient mass transport in the growth plane, MOCVD growth of nitride nanowires requires the introduction of foreign metal catalyst nanoparticles for nucleation, to offer preferential sites for 1D growth. Though the crystalline nature of such metal nanoparticle catalyzed nanowires has been demonstrated to be excellent, the presence of non-native metal catalysts is an undesirable feature.

On the other hand, catalyst-free growth of nitride nanowires has been recently demonstrated by MBE. Typically, AlN buffer layers [6] are used prior to the growth of nitride nanowires. This requires the removal of the nanowires from the underlying substrate post-growth before the fabrication of any electronic/optical devices, since AlN with a bandgap of $6.2 \mathrm{eV}$ (the highest among all known semiconductors) is a very effective barrier for both electron and hole flow between the substrate and the nanowire "active" layers.

The work presented in this paper uses a different nucleation technique utilizing metal droplets of the group III Ga-metal as seeds for III-V nanowire growth by MBE [7]. This method is a contamination free method of growth since no outside elements are introduced into the growth system. In addition, the removal of the AlN nucleation layer paves the way for efficient electron/hole transfer between the nanowires and the underlying substrate. This paper includes experimental data and discussion on MBE growth, structural, and optical characterization of $\mathrm{GaN}$ and InGaN nanowires on p-type $\mathrm{Si}$ (111) substrates by using the novel Ga-droplet nucleation. Though p-type $\mathrm{GaN}$ substrates would be preferable as the substrate material for electrical injection of holes into the nanowires, the p-type Si substrates are chosen at this stage for two reasons: a) cost, and b) it is possible to inject holes into the nanowires using the p-type substrate, in spite of the heterojunction valence band offset, as has been demonstrated in [8,9].

\section{EXPERIMENT}

Nanowire growths were performed in a Veeco GEN 930 MBE system with an idle background pressure of $10^{-10}$ Torr. High-purity solid source effusion cells were used for thermally evaporating $\mathrm{Ga}$ and In with highly controlled fluxes. High-purity $\mathrm{N}_{2}$ gas was passed through a Unibulb RF plasma source (Veeco), and the net active $\mathrm{N}_{2}$ flux was controlled by the $\mathrm{RF}$ power through a computer-controlled matching network controller. The $\mathrm{N}_{2}$ flow rate was controlled for a net background pressure of $2 \times 10^{-5}$ Torr, as measured by an ion-gauge in the growth chamber. Growths were performed on p-type Si (111) substrates after removal of native oxide by a 90 second buffered HF (10:1) dip. The substrates were mounted on 3-inch (100) lapped Si wafers using melted In as an adhesive. Prior to the introduction of the substrates into the growth chamber, they were thoroughly degassed to get rid of water vapor and other atmospheric contaminants adsorbed on the surface: first overnight in a load-lock chamber at 200 ${ }^{\circ} \mathrm{C}$, and then at $400{ }^{\circ} \mathrm{C}$ in a buffer chamber bakeout. After loading into the growth chamber, the $\mathrm{Si}$ (111) substrates were further outgassed at $750{ }^{\circ} \mathrm{C}$ for 15 minutes followed by at $850{ }^{\circ} \mathrm{C}$ for 10 minutes for the removal of additional adsorbents and to offer a clean crystalline surface (as seen by Reflection High-Energy Electron Diffraction - RHEED patterns) for the nucleation of nitride nanowires.

After the growths, the samples were imaged by Scanning Electron Microscopy (SEM) imaging using a Hitachi S-4500 system. TEM imaging was carried out on a JEOL 2010 system using an accelerating voltage of $200 \mathrm{kV}$. Optical characterization of the $\mathrm{GaN}$ and $\mathrm{InGaN}$ nanowires was performed by photoluminescence measurements. The samples were loaded into a 
$\mathrm{LN}_{2}$-cooled cryostat (temperature control range: $80 \mathrm{~K}-300 \mathrm{~K}$ ) and the PL spectra was obtained upon excitation by a $325 \mathrm{~nm} \mathrm{He}-\mathrm{Cd}$ laser. For future work on single nanowires (electrical and optical characterization), we have also observed that they can be 'broken-off' the underlying substrates and suspended into organic solutions by simple ultrasonication [5]. The results of the growth and characterization are described in the rest of this paper.

\section{DISCUSSION}

The initial step for growth consisted of depositing Ga droplets at a substrate temperature of $530{ }^{\circ} \mathrm{C}$ using a Ga Beam Equivalent Pressure (BEP) ranging from $2-5 \times 10^{-8}$ Torr for $25-40$ seconds. Upon deposition, the Ga effusion cell shutter was closed and the sample was exposed to a $\mathrm{N}_{2}$ plasma of 450 Watts for one minute. Following this procedure for the formation of $\mathrm{GaN}$ dots on the surface, the substrate temperature was raised to the desired temperature for nanowire growth and the appropriate cells were opened. Nanowires composed of three different materials have been grown to date $-\mathrm{GaN}, \mathrm{InGaN}$, and $\mathrm{AlGaN} / \mathrm{GaN}$ superlattices. From the preliminary characterization of the superlattice samples, heterostructure of the nanowires along the growth direction can be created by modulating the respective metal fluxes and growth temperatures, offering a substantial degree of freedom for the exploration of various novel bandgap-engineered device architectures. However, the superlattice nanowire samples are in the process of being characterized comprehensively. Therefore, in the rest of this paper, we discuss the growth, structural, and optical characterization results of the $\mathrm{GaN}$ and $\mathrm{InGaN}$ nanowires.

The composition, shape, and density of the $\mathrm{GaN}$ and $\mathrm{InGaN}$ nanowires are strongly dependent on the MBE growth conditions. It has been found that by increasing the deposition time of the initial Ga droplets at a BEP of $2 \times 10^{-8}$ Torr at $530{ }^{\circ} \mathrm{C}$ from 25 to 40 seconds, while keeping the flux constant, the nanowire areal density is increased. This agrees with intuition; as the deposition time is increased, more Ga atoms impinge on the surface, diffuse, form more droplets, which act as nucleation sites for the GaN nanowires. Increasing the Gallium droplet deposition time will continue to increase nanowire nucleation site density until the point is reached where the Gallium drops merge together forming a continuous 2D film.

Figure 1 shows the side view SEM images of four GaN nanowire samples. Nucleation for all four samples occurred with a $2 \times 10^{-8}$ Torr Gallium BEP for 40 seconds at $530{ }^{\circ} \mathrm{C}$ followed by a 1 minute exposure to $450 \mathrm{~W}$ Nitrogen RF Plasma. Following the RF exposure, the substrate temperature was raised to $830{ }^{\circ} \mathrm{C}$ for nanowire growth. Samples 1 (a) and (b) were grown using a Ga BEP of $2 \times 10^{-8}$ Torr. Sample 1(a) was grown for 2 hours, and 1(b) was grown for 3 hours. The growth at this low Ga-flux is sparse (nanowires grow far apart), and a large degree of nonuniformity in the growth rate over the sample can be observed. In addition, though the wires tend to grow in a general vertical direction away from the Si substrate, there is a considerable spread in the tilt angles. An average growth rate of $\sim 200-240 \mathrm{~nm} / \mathrm{hr}$ can be estimated from the lengths, and diameters of the nanowires range from $\sim 20-40 \mathrm{~nm}$. However, the tilt, non-uniformity in the nanowire lengths, and sparsity of wires was considerably reduced by increasing the Ga-flux. Figures 1(c) and (d) show the SEM images of wires grown at a Ga-BEP of $\sim 10^{-7}$ Torr - sample (c) is the result of a 2 hour growth, and (d) was grown for 4 hours. The higher Ga-flux yielded vertical growth rates of $\sim 250 \mathrm{~nm} / \mathrm{hr}$, which is considerably more uniform over the sample. In addition, the tilt was reduced as indicated in Fig 1(d), and the density of nanowires was considerably enhanced. Though the nanowires are separate from each other, the density in 
samples (c) and (d) is high enough such that it is not possible to see through them by SEM, as is possible in (a) and (b). The dependence of the nanowire growths on the Ga flux and the growth time leads us to draw two conclusions: a) that the vertical growth rate is relatively independent of Ga flux, and b) that changes in the Ga-flux changes the area density of the nanowires, not their lengths. This might seem counterintuitive, since the growth is carried out at a high V/III ratio; however, the experimental results indicate that instead of growing longer nanowires at high $\mathrm{Ga}$ fluxes, there is growth of more nanowires of the same length. At a constant growth temperature, there exists a limiting rate at which Ga can be consumed by individual growing wires; the excess metal diffuses along the substrate, coalesces, and seeds more nanowires. A suitable model for this form of growth kinetics would be attractive, and is currently being investigated.

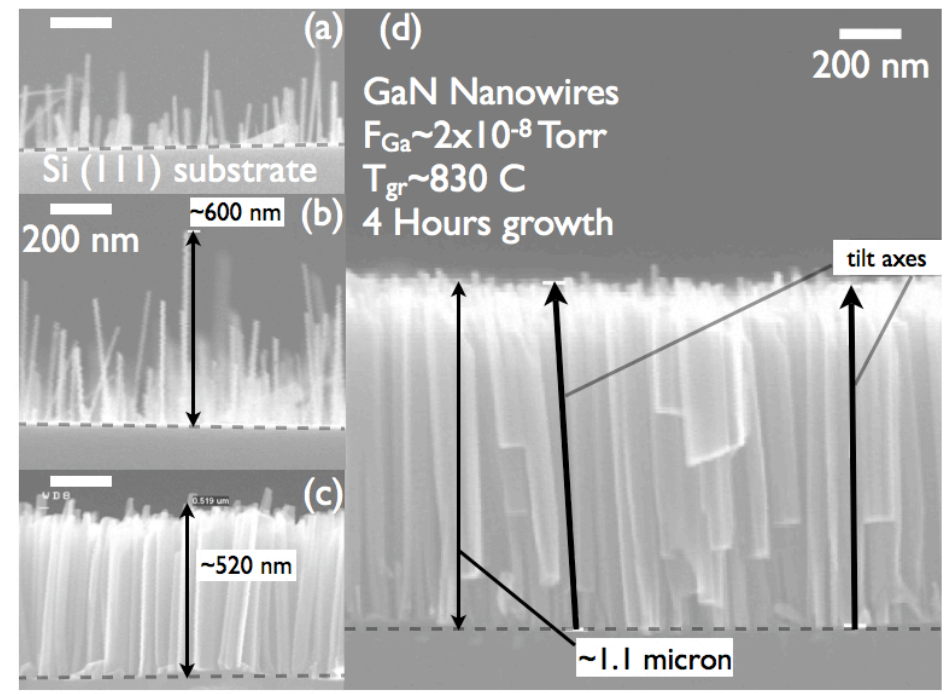

Figure 1. Side view SEM images of MBE-grown GaN nanowires on Si (111) substrates. All figures are of the same scale for ease of comparison. Figures (a) \& (b) were grown with a Ga BEP of $2 \times 10^{-8}$ Torr, while (c) \& (d) were grown with a Ga BEP of $10^{-7}$ Torr. The increased Ga flux leads to a increase in the areal density of nanowires, better alignment, and relatively uniform growth rate over the wafer. The growth rate is $\sim 250 \mathrm{~nm} / \mathrm{hr}$.

Photoluminescence measurements were taken on the GaN nanowire samples on the $\mathrm{Si}$ substrates, and are plotted in Figure 2. Measurements from $80 \mathrm{~K}$ to room temperature show a GaN peak at $\sim 362 \mathrm{~nm}$ at $290 \mathrm{~K}$, which blue shifts to $\sim 358 \mathrm{~nm}$ at $80 \mathrm{~K}$. One inset of Figure 2 shows an SEM image of a GaN nanowire broken off from the Si substrate by sonication. The second inset shows a high-resolution TEM image of a GaN nanowire. The lattice fringes are clearly discernible, and a c-lattice constant of $0.513 \mathrm{~nm}$ is measured, indicating strain-free GaN. An additional piece of vital information from the TEM image is the fact that the growth occurs along the (0001) direction of the GaN wurtzite crystal, which implies that effects of electronic polarization should be available for device engineering. Though relaxed wires would imply the absence of piezoelectric polarization, the spontaneous component of polarization can provide bound charges and large fields in heterostructures grown along the nanowire axis. The photoluminescence data proves the nanowires are entirely composed of GaN, thus validating the completion of the first goal, namely to grow GaN nanowires. The TEM image backs the data from the PL signal and provides information about the strain state of the wires. 


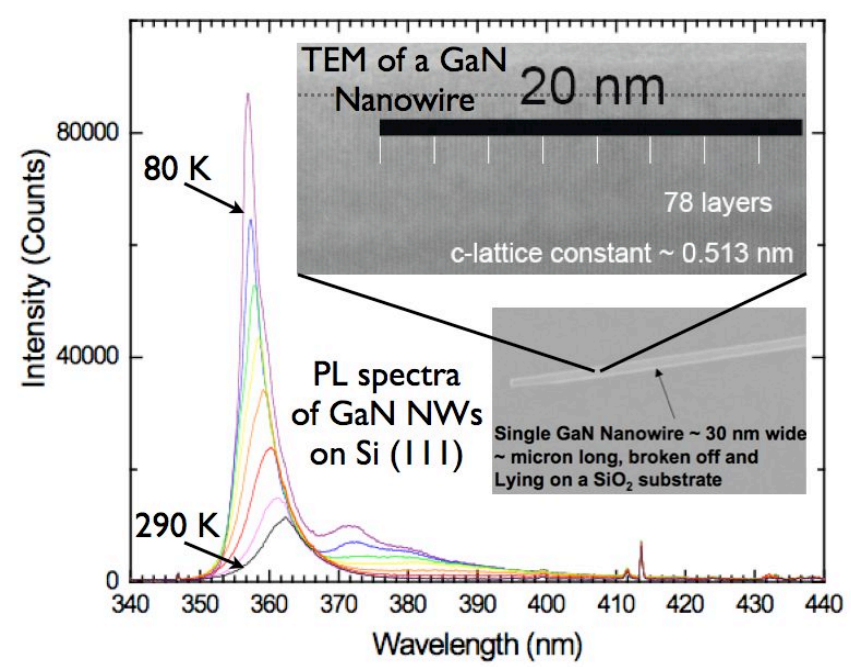

Figure 2. PL spectrum of GaN nanowires on Si (111) substrate. Inset shows SEM of a harvested $\mathrm{GaN}$ wire. Adjacent TEM image verifies GaN c-plane lattice constant and indicates growth along the 0001 direction.

Once GaN nanowires were successfully grown, attention was switched to incorporating Indium into the nanowires to red shift the emission into the green wavelength window $(\sim 520$ $580 \mathrm{~nm})$. Since the energy of the In-N bond is much lower than the Ga-N bond, In does not incorporate into the nanowires at the high temperatures $\left(\sim 830{ }^{\circ} \mathrm{C}\right)$ that the $\mathrm{GaN}$ wires were grown. It was therefore necessary to experiment with growth temperatures that not only allowed the growth of InGaN nanowires, but that were also low enough to allow for significant Indium composition to achieve emission in the green spectral window. The growth of InGaN nanowires proceeded as follows. GaN islands were formed at $530{ }^{\circ} \mathrm{C}$ by depositing Ga for 40 seconds at a BEP of $2 \times 10^{-8}$ Torr, following with one minute exposure to a $450 \mathrm{~W} \mathrm{RF} \mathrm{N}_{2}$ plasma. GaN wires were grown for 30 minutes at a substrate temperature of $830{ }^{\circ} \mathrm{C}$ using a Gallium BEP of $10^{-7}$ Torr and plasma power of $450 \mathrm{~W}$ - this procedure was kept identical to that used earlier for $\mathrm{GaN}$ wires. Following this, the substrate temperature was lowered systematically over a series of samples, the Ga BEP was lowered to $2.25 \times 10^{-8}$ Torr, and the Indium shutter was opened at a BEP of $7.5 \times 10^{-8}$ Torr. InGaN nanowire growth was performed for 90 minutes. The InGaN growth morphology showed a very strong temperature dependence. At a low growth temperature of $550{ }^{\circ} \mathrm{C}$, the InGaN formed a clustered, almost coalesced layer on top of the GaN wires. At a growth temperature of $600{ }^{\circ} \mathrm{C}$, the InGaN started out as wires conformal to the $\mathrm{GaN}$ nanowires under them, but their radii increased, forming golf-tee patterns. However, for temperatures of $650{ }^{\circ} \mathrm{C}$ InGaN nanowire growth occurs with no coalescing of the wires as can be seen in the insert of Figure 3.

Figure 3 shows the PL spectra measured for an InGaN sample grown at $650{ }^{\circ} \mathrm{C}$. On the other hand, InGaN growths at $750{ }^{\circ} \mathrm{C}$ yield PL peaks at $360 \mathrm{~nm}$ which implies that no In was incorporated into the wires, which are thus GaN. Growths at $650 \mathrm{C}$ allowed for In incorporation nominally up to 38\%, giving an effective InGaN PL peak emission at $520 \mathrm{~nm}$ as seen in Figure 3. However, this emission could possibly result from defect states in the bandgap of GaN nanowires, though the likelihood of this is low since no GaN peak is observed in the PL spectra of Fig 3. Further structural evaluation (XRD \& TEM) is being performed for concrete proof of the Indium content of the nanowires. 


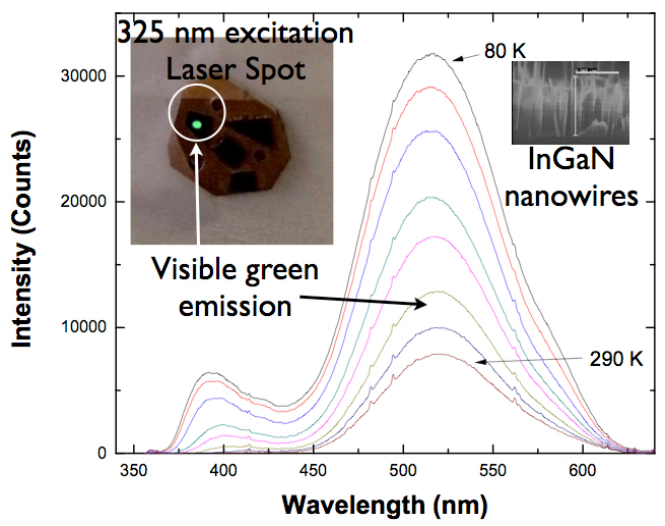

Figure 3. PL data of InGaN nanowires on (111) Si substrate grown at $650{ }^{\circ} \mathrm{C}$. Insets; a picture of $325 \mathrm{~nm}$ laser incident on the sample luminecing green, SEM image of wires grown at $650{ }^{\circ} \mathrm{C}$.

\section{CONCLUSIONS AND FUTURE WORK}

The successful growth of InGaN nanowires, and the identification of the growth conditions necessary to achieve it is an important first step towards various applications. By increasing the In composition, it should be possible to move into the red and the IR regimes of the electromagnetic spectrum, opening up applications not only in optical emitters, but also in photodetectors, photovoltaics, and field-emission devices and novel sensor technologies. Imminent work includes using p-type Silicon substrates as a hole injector to Silicon doped InGaN nanowires for green wavelength emission [9].

\section{ACKNOWLEDGMENTS}

The authors would like to thank an NSF CAREER award (DMR-0645698) and Dr. Chagaan Baatar from Office of Naval Research for financial support and helpul discussions.

\section{REFERENCES}

1. L. Lave, MRS Bulletin. 33, (April 2008).

2. S. Muthu, F. Schuurman, M. Pashley, IEEE Journal on Selected Topics in Quantum Electronics. 8, No. 2 (2002).

3. K. Bertness, N. Sanford, A. Davydov, The Journal of Defense Software Engineering. (2006).

4. F. Qian, Y. Li, S. Gradecak, D. Wang, C. Barrelet, C. Lieber. Nano Letters. 4, No. $101975-$ 1979 (2004).

5. K. Bertness, N. Sanford, J. Barker, J. Schlager, A. Roshko, A. Davydov, I. Levin. J. of Electronic Materials. 35, No. 4 576-580 (April 2008).

6. K. Bertness, A. Roshko, N. Sanford, J. Barker, A. Davydov, Journal of Crystal Growth. 287, 522-527 (2006).

7. A. Kikuchi, M. Kawai, M. Tada, K. Kishino, Japanese Journal of Applied Physics. 43, 12A L1524-L1526 (2004).

8. M. A. Zimmler, J. Bao, I. Shalish, W. Yi, V. Narayanmurti, and F. Capasso, Nanotechnology, 18, 395201 (2007).

9. X. Sun, J. Huang, J. Wang, Z. Xu. Nano Letters. 8, No. 4 1219-1223 (2008). 